\title{
APPROXIMATE ANTILINEAR EIGENVALUE PROBLEMS AND RELATED INEQUALITIES
}

\author{
STEPHAN RAMON GARCIA
}

(Communicated by Joseph A. Ball)

\begin{abstract}
If $T$ is a complex symmetric operator on a separable complex Hilbert space $\mathcal{H}$, then the spectrum $\sigma(|T|)$ of $\sqrt{T^{*} T}$ can be characterized in terms of a certain approximate antilinear eigenvalue problem. This approach leads to a general inequality (applicable to any bounded operator $T: \mathcal{H} \rightarrow \mathcal{H}$ ), in terms of the spectra of the selfadjoint operators $\operatorname{Re} T$ and $\operatorname{Im} T$, restricting the possible location of elements of $\sigma(|T|)$. A sharp inequality for the operator norm is produced, and the extremal operators are shown to be complex symmetric.
\end{abstract}

\section{INTRODUCTION}

A classical result in matrix theory asserts that the singular values of an $n \times n$ complex symmetric matrix $A$ can be characterized as the nonnegative solutions $\lambda$ to the antilinear eigenvalue problem $A \mathbf{x}=\lambda \overline{\mathbf{x}}$, where $\mathbf{x} \neq \mathbf{0}$ and $\overline{\mathbf{x}}$ denotes entry-byentry complex conjugation of a vector $\mathbf{x} \in \mathbb{C}^{n}[13$, Sect. 4.4]. A similar antilinear eigenvalue problem also characterizes the singular values of complex symmetric operators, a relatively large class that includes many standard and well-studied examples (see Section 2 for background and examples).

In this note, we establish that the spectrum $\sigma(|T|)$ of the modulus $|T|=\sqrt{T^{*} T}$ of a complex symmetric operator $T: \mathcal{H} \rightarrow \mathcal{H}$ (acting on a separable complex Hilbert space $\mathcal{H}$ ) can be characterized in terms of a certain approximate antilinear eigenvalue problem (Theorem 2 ). We use this approach, along with a complex symmetric block matrix technique, to derive a system of general inequalities restricting the possible location of spectral values $\lambda \in \sigma(|T|)$ for an arbitrary bounded operator $T: \mathcal{H} \rightarrow \mathcal{H}$ (Theorem 1). Finally, we note that a special case of our main inequality yields a sharp inequality for the operator norm for which the extremal operators are complex symmetric.

It is well known that the operator norm of a bounded linear operator $T: \mathcal{H} \rightarrow \mathcal{H}$ coincides with the supremum of the spectrum $\sigma(|T|)$ of the positive operator $|T|=$ $\sqrt{T^{*} T}$. Moreover, the eigenvalues of $|T|$ (i.e., the singular values of $T$ ) are important in many applications. Our first theorem provides geometric information on the

Received by the editors September 11, 2006 and, in revised form, September 28, 2006.

2000 Mathematics Subject Classification. Primary 47A30.

Key words and phrases. Complex symmetric operator, operator norm, triangle inequality, selfadjoint operator, Cartesian decomposition, approximate antilinear eigenvalue problem, antilinear, spectrum.

This work was partially supported by National Science Foundation Grant DMS-0638789. 
possible location of elements of $\sigma(|T|)$ in terms of the spectra of the selfadjoint components of $T$.

Theorem 1. Let $T: \mathcal{H} \rightarrow \mathcal{H}$ be a bounded linear operator on a separable complex Hilbert space $\mathcal{H}$ and let $T=A+i B$ where $A$ and $B$ are selfadjoint. Every $\lambda \in \sigma(|T|)$ satisfies the inequality

$$
\inf _{a \in \sigma(A)}|a+\lambda \cos \theta| \inf _{a \in \sigma(A)}|a-\lambda \cos \theta| \leq \sup _{b \in \sigma(B)}|b+\lambda \sin \theta| \sup _{b \in \sigma(B)}|b-\lambda \sin \theta|
$$

for all real $\theta$ such that there exists a real sequence $\theta_{n} \rightarrow \theta$ such that $\pm \lambda \cos \theta_{n}$ belongs to the resolvent set $\rho(A)$ of $A$.

We remark that the additional parameter $\theta$ might provide some flexibility in particular applications and that the seemingly complicated restrictions on this parameter are usually trivial in practice. For example, the preceding theorem easily implies the following corollary:

Corollary 1. Let $T$ denote an $n \times n$ matrix with complex entries and let $T=A+i B$ where $A$ and $B$ are selfadjoint. Every eigenvalue $\lambda$ of the positive matrix $T=\sqrt{T^{*} T}$ satisfies the inequality

$$
\min _{a \in \sigma(A)}|a+\lambda \cos \theta| \min _{a \in \sigma(A)}|a-\lambda \cos \theta| \leq \max _{b \in \sigma(B)}|b+\lambda \sin \theta| \max _{b \in \sigma(B)}|b-\lambda \sin \theta|
$$

for all real $\theta$.

The proof of Theorem 1 relies on a recent factorization theorem (Lemma 1) for the class of complex symmetric operators. We therefore require a few preliminary remarks before we begin the proof.

\section{Complex symmetric operators}

Suppose that $\mathcal{H}$ is a separable complex Hilbert space endowed with a conjugation $C: \mathcal{H} \rightarrow \mathcal{H}$. Specifically, this means that $C$ is an antilinear (i.e., conjugate linear) operator on $\mathcal{H}$ that is involutive $\left(C^{2}=I\right)$ and isometric, meaning that $\langle x, y\rangle=$ $\langle C y, C x\rangle$ holds for all $x, y$ in $\mathcal{H}$. A bounded operator $T: \mathcal{H} \rightarrow \mathcal{H}$ is called $C$ symmetric if $T=C T^{*} C$ and complex symmetric if it is $C$-symmetric with respect to some conjugation $C$.

For a fixed conjugation $C$, there exists an orthonormal basis $\left(e_{n}\right)_{n=1}^{\operatorname{dim} \mathcal{H}}$ of $\mathcal{H}$ such that $C e_{n}=e_{n}$ for all $n$ [9, Lem. 1]. We refer to such a basis as a $C$ real orthonormal basis and note that the matrix representation of a $C$-symmetric operator with respect to such a basis is symmetric (see [9, Prop. 2] or [8, Sect. 2.4]). In particular, an operator $T$ is complex symmetric if and only if it is unitarily equivalent to a symmetric matrix with complex entries, considered as an operator on an $l^{2}$ space of the appropriate dimension.

The class of complex symmetric operators contains many standard and wellknown operators. Consider the following examples:

Example 1. Operators on $\mathbb{C}^{n}$ induced by symmetric matrices with complex entries are complex symmetric (the corresponding conjugation $C$ being entry-byentry complex conjugation). The study of such matrices has deep classical roots $[15,20,23,24,25]$ and the connection between complex symmetric matrices and univalent function theory has long been known (see [5, Sect. 4.4] and [6]). Complex symmetric matrices also appear in the consideration of quantum reaction dynamics [2], the numerical simulation of high-voltage insulators [19], and thermoelastic 
wave propagation [22]. They have also been the focus of recent algorithmic work (see $[1,12,14]$, for example). The basic theory of complex symmetric matrices is discussed in the texts $[7,13]$.

Example 2. Since a Hankel matrix is symmetric, any (finite or infinite) Hankel matrix defines a complex symmetric operator on its associated $l^{2}$ space. Moreover, Hankel operators on Hardy spaces enjoy a prominent role in mathematical systems theory (see the recent texts $[16,17]$ ).

Example 3. All normal operators are complex symmetric. Indeed, if $M_{z}$ denotes the operator of multiplication by the independent variable on a Lebesgue space $L^{2}(\mu)$ (where $\mu$ is a compactly supported Borel measure on $\mathbb{C}$ ), then $M_{z}=C M_{z}^{*} C$ where $C$ denotes complex conjugation on $L^{2}(\mu)$. The spectral theorem asserts that any normal operator can be represented as a direct sum of such operators, and hence any normal operator is complex symmetric.

Example 4. Every $n \times n$ Toeplitz matrix induces a complex symmetric operator on $\mathbb{C}^{n}$. Indeed, this simply reflects the symmetry of a Toeplitz matrix with respect to the counter-diagonal. More generally, all compressed Toeplitz operators on the Hardy space $H^{2}$ are complex symmetric $[8,9,10]$. This includes all (scalar) Jordan models operators (often called compressed shifts), the natural infinite-dimensional counterparts of the familiar Jordan blocks from linear algebra (see the texts $[3,16]$ for background). We should also mention the recent paper [4], which characterizes complex symmetric contractions in terms of their characteristic functions. In particular, an explicit description of all complex symmetric contractions with defect index 1 or 2 is now known.

Example 5. Another family of examples is furnished by considering integral operators whose kernels possess certain functional symmetries. For example, the Volterra integration operator

$$
[V f](x)=\int_{0}^{x} f(t) d t
$$

on $L^{2}[0,1]$ is $C$-symmetric with respect to $[C f](x)=\overline{f(1-x)}$.

Although there are many other examples, we restrict ourselves here to these few in order to proceed to the matter at hand.

\section{Approximate Antilinear eigenvalue problems}

Recall that the polar decomposition $T=U|T|$ of an operator $T: \mathcal{H} \rightarrow \mathcal{H}$ expresses $T$ uniquely as the product of a positive operator $|T|=\sqrt{T^{*} T}$ and a partial isometry $U$ which satisfies $\operatorname{ker} U=\operatorname{ker}|T|$ and maps cl ran $|T|$ onto $\operatorname{cl} \operatorname{ran} T$. If $T$ is a $C$-symmetric operator, then we can decompose the partial isometry $U$ as the product of $C$ with a partial conjugation. We say that an antilinear operator $J$ is a partial conjugation if $J$ restricts to a conjugation on $(\operatorname{ker} J)^{\perp}$ (with values in the same space). In particular, the linear operator $J^{2}$ is the orthogonal projection onto the closed subspace $\operatorname{ran} J=(\operatorname{ker} J)^{\perp}$.

The following lemma, whose proof we briefly sketch, is from [10]:

Lemma 1. If $T: \mathcal{H} \rightarrow \mathcal{H}$ is a bounded $C$-symmetric operator, then $T=C J|T|$, where $J$ is a partial conjugation, supported on $\operatorname{cl} \operatorname{ran}|T|$, which commutes with $|T|=$ $\sqrt{T^{*} T}$. 
Proof. Write the polar decomposition $T=U|T|$ of $T$ and note that $T=C T^{*} C=$ $C|T| U^{*} C=\left(C U^{*} C\right)\left(C U|T| U^{*} C\right)$ since $U^{*} U$ is the orthogonal projection onto cl ran $|T|$. One shows that $\operatorname{ker} C U^{*} C=\operatorname{ker} C U|T| U^{*} C$, notes that $C U^{*} C$ is a partial isometry and that $C U|T| U^{*} C$ is positive, then concludes from the uniqueness of the terms in the polar decomposition that $U=C U^{*} C$ (so that $U$ is $C$-symmetric) and that the antilinear operator $J=C U=U^{*} C$ commutes with $|T|$. One then verifies that $J$ is a partial conjugation supported on $\operatorname{cl} \operatorname{ran}|T|$.

Now recall that Weyl's criterion [18, Thm. VII.12] states that if $A$ is a bounded selfadjoint operator, then $\lambda \in \sigma(A)$ if and only if there exists a sequence $f_{n}$ of unit vectors so that $\lim _{n \rightarrow \infty}\left\|(A-\lambda I) f_{n}\right\|=0$. The following theorem characterizes $\sigma(|T|)$ in terms of what one might call an approximate antilinear eigenvalue problem:

Theorem 2. If $T$ is a bounded $C$-symmetric operator and $\lambda \geq 0$, then

(i) $\lambda$ belongs to $\sigma(|T|)$ if and only if there exists a sequence of unit vectors $f_{n}$ such that $\lim _{n \rightarrow \infty}\left\|(T-\lambda C) f_{n}\right\|=0$. Moreover, the $f_{n}$ may be chosen so that $J f_{n}=f_{n}$ for all $n$ (here $J$ denotes the partial conjugation from Lemma 1).

(ii) $\lambda$ is an eigenvalue of $|T|$ (i.e., a singular value of $T$ ) if and only if the antilinear eigenvalue problem $T f=\lambda C f$ has a nonzero solution $f$. Moreover, $f$ may be chosen so that $J f=f$.

Proof. The second assertion of the theorem, which follows easily from the first, is proved in [10], and hence only the first assertion requires proof. Without loss of generality, we may assume that the partial conjugation $J$ in the factorization $T=C J|T|$ of Lemma 1 is a conjugation on all of $\mathcal{H}$ since we need only add a partial conjugation supported on the complementary space ker $|T|$ to obtain a conjugation on all of $\mathcal{H}$ which agrees with $J$ on $\operatorname{cl} \operatorname{ran}|T|$.

By Weyl's criterion, $\lambda \geq 0$ belongs to $\sigma(|T|)$ if and only if there exists a sequence $u_{n}$ of unit vectors so that $\left\||T| u_{n}-\lambda u_{n}\right\| \rightarrow 0$. Since $J$ is isometric and commutes with $|T|$, this happens if and only if $\left\||T| J u_{n}-\lambda J u_{n}\right\| \rightarrow 0$ as well. Since not both of $\frac{1}{2}\left(u_{n}+J u_{n}\right)$ and $\frac{1}{2 i}\left(u_{n}-J u_{n}\right)$ can be zero for a given $n$, we can obtain a sequence of unit vectors $f_{n}$ with the property that $J f_{n}=f_{n}$ and that $\left\|(T-\lambda C) f_{n}\right\|=$ $\left\|C T f_{n}-\lambda f_{n}\right\|=\left\|J|T| f_{n}-\lambda f_{n}\right\|=\left\||T| f_{n}-\lambda f_{n}\right\|$ tends to 0 . On the other hand, if a sequence $f_{n}$ satisfying (i) exists, then it follows from Lemma 1 that $\lim _{n \rightarrow \infty}\left\|(|T|-\lambda) f_{n}\right\|=0$. By Weyl's criterion, $\lambda \in \sigma(|T|)$.

We are now ready to begin the proof of Theorem 1 .

\section{Proof of Theorem 1}

The proof of Theorem 1 consists of two main parts. First, we prove the theorem in the special case where $T$ is a complex symmetric operator. The proof of the general case will follow from the complex symmetric case via a block matrix argument.

Part I: Assume that $T$ is a complex symmetric operator. In other words, there exists a conjugation $C: \mathcal{H} \rightarrow \mathcal{H}$ so that $T=C T^{*} C$. By Theorem 2, a real number $\lambda \geq 0$ belongs to $\sigma(|T|)$ if and only if there exists a sequence of unit vectors $f_{n}$ such that $\left\|T f_{n}-\lambda C f_{n}\right\| \rightarrow 0$. Given a real number $\theta$, write $f_{n}=e^{-i \theta / 2} g_{n}$ and note that (since $C$ is antilinear) the unit vectors $g_{n}$ have the property that $\left\|T g_{n}-e^{i \theta} \lambda C g_{n}\right\| \rightarrow 0$. We next decompose the $g_{n}$ into their $C$-real and $C$-imaginary 
parts and write $g_{n}=x_{n}+i y_{n}$, where $C x_{n}=x_{n}$ and $C y_{n}=y_{n}$ for all $n$. Upon expanding $T g_{n}-e^{i \theta} \lambda C g_{n}$ it follows that

$$
\left[(A-\lambda \cos \theta) x_{n}-(B+\lambda \sin \theta) y_{n}\right]+i\left[(B-\lambda \sin \theta) x_{n}+(A+\lambda \cos \theta) y_{n}\right]
$$

tends to zero. In particular, the $C$-real and $C$-imaginary components of $(2)$ both tend to zero separately. We therefore see that

$$
\begin{aligned}
& (A-\lambda \cos \theta) x_{n}-(B+\lambda \sin \theta) y_{n} \rightarrow 0, \\
& (B-\lambda \sin \theta) x_{n}+(A+\lambda \cos \theta) y_{n} \rightarrow 0 .
\end{aligned}
$$

Now observe that we may assume that the operators $A \pm \lambda \cos \theta$ are both invertible. Indeed, the hypothesis of the theorem asserts that there is a sequence $\theta_{n} \rightarrow \theta$ such that the operators $A \pm \lambda \cos \theta_{n}$ are invertible. If we establish the desired inequality (1) with $\theta_{n}$ in place of $\theta$, then (1) will follow after passing to the limit. If the operators $A \pm \lambda \cos \theta$ are both invertible, then we derive from (3) and (4) that

$$
\left[I+(A+\lambda \cos \theta)^{-1}(B-\lambda \sin \theta)(A-\lambda \cos \theta)^{-1}(B+\lambda \sin \theta)\right] y_{n} \rightarrow 0 .
$$

There are two possible cases:

CASE 1: If $y_{n} \rightarrow 0$, then $\left\|x_{n}\right\| \rightarrow 1$ and, since $\left\|T g_{n}-e^{i \theta} \lambda C g_{n}\right\| \rightarrow 0$, we find that $\left\|T x_{n}-e^{i \theta} \lambda x_{n}\right\|$ also tends to zero. Since $T=C T^{*} C$ and $C x_{n}=x_{n}$ for all $n$, it also follows that $\left\|T^{*} x_{n}-e^{-i \theta} \lambda x_{n}\right\| \rightarrow 0$. However, this would imply that

$$
\begin{aligned}
\left\|(A-\lambda \cos \theta) \frac{x_{n}}{\left\|x_{n}\right\|}\right\| & =\frac{1}{\left\|x_{n}\right\|}\left\|\frac{1}{2}\left(T+T^{*}\right) x_{n}-\frac{1}{2}\left(e^{i \theta}+e^{-i \theta}\right) \lambda x_{n}\right\| \\
& \leq \frac{1}{2\left\|x_{n}\right\|}\left\|T x_{n}-e^{i \theta} \lambda x_{n}\right\|+\frac{1}{2\left\|x_{n}\right\|}\left\|T^{*} x_{n}-e^{-i \theta} \lambda x_{n}\right\|
\end{aligned}
$$

tends to zero as well, contradicting the assumption that $A-\lambda \cos \theta$ was invertible.

CASE 2: If $y_{n}$ does not tend to zero, then there exists a subsequence of $y_{n}$ that is bounded away from zero. It therefore follows from (5) that the operator

$$
I+(A+\lambda \cos \theta)^{-1}(B-\lambda \sin \theta)(A-\lambda \cos \theta)^{-1}(B+\lambda \sin \theta)
$$

is not invertible and hence

$$
\begin{aligned}
1 & \leq\left\|(A+\lambda \cos \theta)^{-1}(B-\lambda \sin \theta)(A-\lambda \cos \theta)^{-1}(B+\lambda \sin \theta)\right\| \\
& \leq\left\|(A+\lambda \cos \theta)^{-1}\right\|\left\|(A-\lambda \cos \theta)^{-1}\right\|\|B+\lambda \sin \theta\|\|B-\lambda \sin \theta\| \\
& \leq \sup _{a \in \sigma(A)}|a+\lambda \cos \theta|^{-1} \sup _{a \in \sigma(A)}|a-\lambda \cos \theta|^{-1} \sup _{b \in \sigma(B)}|b+\lambda \sin \theta| \sup _{b \in \sigma(B)}|b-\lambda \sin \theta| .
\end{aligned}
$$

Rearranging the preceding inequality yields (1).

Part II: Now suppose that $T$ is a bounded (not necessarily complex symmetric) operator and let $T=A+i B$, where $A$ and $B$ are both selfadjoint operators. If $C$ is any conjugation on $\mathcal{H}$, then it is easily verified that the operator $\widetilde{T}$ on $\mathcal{H} \oplus \mathcal{H}$ defined by

$$
\widetilde{T}=\left(\begin{array}{cc}
T & 0 \\
0 & C T^{*} C
\end{array}\right)
$$

is a $\widetilde{C}$-symmetric extension of $T$. Here $\widetilde{C}$ denotes the conjugation

$$
\widetilde{C}=\left(\begin{array}{cc}
0 & C \\
C & 0
\end{array}\right)
$$


acting on $\mathcal{H} \oplus \mathcal{H}$. Decomposing $\widetilde{T}$ into its real and imaginary components, we find that $\widetilde{T}=\widetilde{A}+i \widetilde{B}$ where the operators $\widetilde{A}$ and $\widetilde{B}$, given by

$$
\widetilde{A}=\left(\begin{array}{cc}
A & 0 \\
0 & C A C
\end{array}\right), \quad \widetilde{B}=\left(\begin{array}{cc}
B & 0 \\
0 & C B C
\end{array}\right),
$$

are selfadjoint, $\widetilde{C}$-symmetric, and satisfy $\sigma(\widetilde{A})=\sigma(A)$ and $\sigma(\widetilde{B})=\sigma(B)$. Since

$$
\widetilde{T}^{*} \widetilde{T}=\left(\begin{array}{cc}
T^{*} T & 0 \\
0 & C T T^{*} C
\end{array}\right)
$$

we also see that the nonzero elements $\sigma(|T|)$ and $\sigma(|\widetilde{T}|)$ are identical (see [11, $\operatorname{Pr} .76])$.

Proceeding as in Part I, we find a sequence of unit vectors $\widetilde{g}_{n}$ in $\mathcal{H} \oplus \mathcal{H}$ such that $\left\|\widetilde{T} \widetilde{g}_{n}-e^{i \theta} \lambda \widetilde{C} \widetilde{g}_{n}\right\| \rightarrow 0$. Since the vectors left fixed by the conjugation $\widetilde{C}$ are precisely those of the form $(x, C x)$ where $x$ is a vector in $\mathcal{H}$, we decompose the $\widetilde{g}_{n}$ in terms of their $\widetilde{C}$-real and $\widetilde{C}$-imaginary components:

$$
\widetilde{g}_{n}=\widetilde{x}_{n}+i \widetilde{y}_{n}=\left(\begin{array}{c}
x_{n} \\
C x_{n}
\end{array}\right)+i\left(\begin{array}{c}
y_{n} \\
C y_{n}
\end{array}\right)
$$

where the $x_{n}$ and $y_{n}$ are not necessarily fixed by the original conjugation $C$. As before, we may assume that the operators $\widetilde{A} \pm \lambda \cos \theta$ are both invertible and reach the analog of (5).

CAse 1: If $\widetilde{y}_{n}$ tends to zero, then as in Case 1 of Part I it follows that $\left\|\widetilde{x}_{n}\right\| \rightarrow 1$ whence $\left\|x_{n}\right\|$ tends to $1 / \sqrt{2}$ and $\left\|\widetilde{T} \widetilde{x}_{n}-e^{i \theta} \lambda \widetilde{x}_{n}\right\| \rightarrow 0$. Since

$$
\left(\begin{array}{cc}
T & 0 \\
0 & C T^{*} C
\end{array}\right)\left(\begin{array}{c}
x_{n} \\
C x_{n}
\end{array}\right)-e^{i \theta} \lambda\left(\begin{array}{c}
x_{n} \\
C x_{n}
\end{array}\right)
$$

tends to zero, it follows that $\left\|T x_{n}-e^{i \theta} \lambda x_{n}\right\|$ and $\left\|T^{*} x_{n}-e^{i \theta} \lambda x_{n}\right\|$ both tend to zero separately. As before, we deduce from this that the operator $\widetilde{A}-\lambda \cos \theta$ (and hence $A-\lambda \cos \theta)$ is not invertible, a contradiction.

CASE 2: Since $\widetilde{y}_{n}$ cannot tend to zero, we can deduce as before that

$$
(\widetilde{A}+\lambda \cos \theta)^{-1}(\widetilde{B}-\lambda \sin \theta)(\widetilde{A}-\lambda \cos \theta)^{-1}(\widetilde{B}+\lambda \sin \theta)
$$

has norm $\geq 1$. Since the selfadjoint operators $\widetilde{A}$ and $\widetilde{B}$ have precisely the same spectra as $A$ and $B$, respectively, we may proceed as we did in Case 2 of Part I to complete the proof.

\section{A SHARP INEQUALITY FOR THE OPERATOR NORM}

We conclude this note with a few special cases of Theorem 1. Although the following estimates are not deep and can be proved by different (admittedly simpler, see below) means, they do illustrate that one can indeed obtain sharp inequalities using Theorem 1. Moreover, we will also observe that complex symmetric operators play the role of extremal operators for these inequalities.

Corollary 2. If $A$ and $B$ are selfadjoint, then

$$
\|A+i B\| \leq \frac{a_{+}-a_{-}}{2}+\sqrt{\left(\frac{a_{+}+a_{-}}{2}\right)^{2}+\|B\|^{2}},
$$


where $a_{+}=\sup \sigma(A)$ and $a_{-}=\inf \sigma(A)$. Given the data $\left\{a_{+}, a_{-},\|B\|\right\}$, the inequality (6) is sharp in the sense that there exists an operator $T=A+i B$ with the given data for which equality holds.

Proof. We may assume that $T=A+i B \neq 0$ so that $\lambda_{+}=\sup \sigma(|T|)=\|T\|>0$. Since $A$ and $B$ are selfadjoint, it is clear that

$$
\|A\|=\sup _{\|x\|=1}|\langle A x, x\rangle| \leq \sup _{\|x\|=1} \sqrt{\langle A x, x\rangle^{2}+\langle B x, x\rangle^{2}}=\sup _{\|x\|=1}|\langle T x, x\rangle| \leq\|T\| .
$$

If either $\left|a_{+}\right|=\lambda_{+}$or $\left|a_{-}\right|=\lambda_{+}$, then

$$
\|T\|=\max \left\{\left|a_{+}\right|,\left|a_{-}\right|\right\}=\frac{1}{2}\left|a_{+}-a_{-}\right|+\frac{1}{2}\left|a_{+}+a_{-}\right|,
$$

whence (6) is automatically satisfied. On the other hand, if $\max \left\{\left|a_{+}\right|,\left|a_{-}\right|\right\}<\lambda_{+}$, then $|a|<\lambda_{+}$holds for each $a$ in $\sigma(A)$. In particular, we see that

$$
\begin{aligned}
& \inf _{a \in \sigma(A)}\left|a+\lambda_{+}\right|=\lambda_{+}+a_{-}, \\
& \inf _{a \in \sigma(A)}\left|a-\lambda_{+}\right|=\lambda_{+}-a_{+} .
\end{aligned}
$$

Since $\sup _{b \in \sigma(B)}|b|=\|B\|$, applying inequality (1) of Theorem 1 with $\theta=0$ we find that $\left(\lambda_{+}+a_{-}\right)\left(\lambda_{+}-a_{+}\right) \leq\|B\|^{2}$. This yields the quadratic inequality

$$
\lambda_{+}^{2}-\left(a_{+}-a_{-}\right) \lambda_{+}-\left(a_{+} a_{-}+\|B\|^{2}\right) \leq 0 .
$$

A straightforward analysis of the polynomial $p(t)=t^{2}-\left(a_{+}-a_{-}\right) t-\left(a_{+} a_{-}+\|B\|^{2}\right)$ yields the inequality (6).

If data $\left\{a_{1}, a_{2}, b\right\}$ is specified (where $a_{1} \geq a_{2}$ and $b \geq 0$ ), then the operator $T: \mathbb{C}^{2} \rightarrow \mathbb{C}^{2}$ defined by the complex symmetric matrix

$$
\left(\begin{array}{cc}
a_{1} & i b \\
i b & a_{2}
\end{array}\right)=\left(\begin{array}{cc}
a_{1} & 0 \\
0 & a_{2}
\end{array}\right)+i\left(\begin{array}{cc}
0 & b \\
b & 0
\end{array}\right)
$$

clearly satisfies $\sigma(A)=\left\{a_{1}, a_{2}\right\}$ (so that $a_{+}=a_{1}$ and $a_{-}=a_{2}$ ) and $\|B\|=b$. A direct computation shows that the norm of $T$ is given by

$$
\frac{a_{1}-a_{2}}{2}+\sqrt{\left(\frac{a_{1}+a_{2}}{2}\right)^{2}+b^{2}} .
$$

The easiest way to verify this is to calculate the eigenvalues of the selfadjoint operator $T^{*} T$ and compare them to the square of the expression above. In particular, this example shows that (6) provides the best possible upper bound on $\|A+i B\|$, given only the data $\left\{a_{+}, a_{-},\|B\|\right\}$.

We should also mention another proof of Corollary 2. Let $\alpha=\frac{1}{2}\left(a_{+}+a_{-}\right)$ and write $A+i B=(A-\alpha I)+(i B+\alpha I)$. It follows from the triangle inequality that $\|A+i B\| \leq\|A-\alpha I\|+\|i B+\alpha I\|$. It is easy to see that the operator $A-\alpha I$ has norm $\frac{1}{2}\left(a_{+}-a_{-}\right)$and that the normal operator $i B+\alpha I$ has norm $\|i B+\alpha I\|=\sqrt{\alpha^{2}+\|B\|^{2}}$. A similar approach even yields the inequality

$$
\|A+i B\| \leq \sqrt{\left(\frac{a_{+}-a_{-}}{2}\right)^{2}+\left(\frac{b_{+}+b_{-}}{2}\right)^{2}}+\sqrt{\left(\frac{a_{+}+a_{-}}{2}\right)^{2}+\left(\frac{b_{+}-b_{-}}{2}\right)^{2}},
$$

which takes into account the additional data $b_{+}=\sup \sigma(B)$ and $b_{-}=\inf \sigma(B)$. 
Example 6. It is well known that the Volterra integration operator

$$
[T f](x)=\int_{0}^{x} f(y) d y
$$

on $L^{2}[0,1]$ has norm equal to $2 / \pi \approx 0.63662$. A straightforward derivation of this fact can be found in [11, Problem 188], and an approach using antilinear eigenvalue problems for complex symmetric operators is discussed in [10]. The Cartesian decomposition of the Volterra operator into its real and imaginary components is

$$
[T f](x)=\left[\frac{1}{2} \int_{0}^{1} f(y) d y\right]+i \frac{1}{2 i}\left[\int_{0}^{x} f(y) d y+\int_{1}^{x} f(y) d y\right] .
$$

In particular, we note that $A$ is a rank-one operator with $\|A\|=a_{+}=1 / 2$ and $a_{-}=0$. Since the imaginary component

$$
[B f](x)=\frac{1}{2 i}\left(\int_{0}^{x} f(y) d y+\int_{1}^{x} f(y) d y\right)
$$

is a compact selfadjoint operator, finding $\sigma(B)$ amounts to a straightforward eigenvalue problem. A routine calculation shows that $\sigma(B)=\left\{((2 n+1) \pi)^{-1}: n \in \mathbb{Z}\right\}$ whence $\|B\|=1 / \pi$. Applying (6) to this data, we find that $\|T\| \leq 0.65475$, which is substantially closer to the true value $\|T\| \approx 0.63662$ than the bound $\|T\| \leq\|A\|+\|B\| \approx 0.81831$ provided from the triangle inequality.

We conclude with another simple corollary. Recall that an operator $T=A+i B$ is called a contraction if $\|T\| \leq 1$. It is not hard to see that $\|A\| \leq 1$ is necessary for $T$ to be a contraction. Equally clear is that if $A$ is a contraction, then the condition $\|B\| \leq 1-\|A\|$ is sufficient for $T$ to be a contraction. One can say a little more:

Corollary 3. If $A$ is a selfadjoint contraction, $a_{+}=\sup \sigma(A)$, and $a_{-}=\sup \sigma(A)$, then $T=A+i B$ is a contraction for any selfadjoint operator $B$ satisfying

$$
\|B\| \leq \sqrt{\left(1-a_{+}\right)\left(1+a_{-}\right)} .
$$

Moreover, the inequality (8) is sharp in the sense that given the data $\left\{a_{+}, a_{-}\right\}$, there exists a selfadjoint operator $A$ such that $a_{+}=\sup \sigma(A)$ and $a_{-}=\inf \sigma(A)$ and a selfadjoint operator $B$ such that $T=A+i B$ is a contraction and $\|B\|=$ $\sqrt{\left(1-a_{+}\right)\left(1+a_{-}\right)}$.

Proof. The upper bound on $\|B\|$ follows from the inequality (6) upon setting the right-hand side of (6) equal to 1 and performing a straightforward computation. That the inequality (8) is sharp follows from the fact that the inequality (6) is sharp. In other words, the same $2 \times 2$ matrices $A$ and $B$ (with $a_{+}=a_{1}, a_{-}=a_{2}$, and $\left.b=\sqrt{\left(1-a_{+}\right)\left(1+a_{-}\right)}\right)$from the proof of Corollary 2 will furnish an example showing that (8) is sharp.

\section{ACKNowledgments}

The author wishes to thank D. Sarason and M. Putinar for their comments and suggestions. 


\section{REFERENCES}

[1] Arbenz, P., Hochstenbach, M.E., A Jacobi-Davidson method for solving complex symmetric eigenvalue problems, SIAM J. Sci. Comput. 25 (2004), 1655-1673. MR2087330 (2005g:65067)

[2] Bar-On, I., Ryaboy, V., Fast diagonalization of large and dense complex symmetric matrices, with applications to quantum reaction dynamics, SIAM J. Sci. Comput. 18 (1997), 1412-1435. MR1465664 (98g:65036)

[3] Bercovici, H., Operator Theory and Arithmetic in $H^{\infty}$, Amer. Math. Soc., Providence, R.I., 1988. MR954383 (90e:47001)

[4] Chevrot, N., Fricain, E., Timotin, D., The characteristic function of a complex symmetric contraction, Proc. Amer. Math. Soc. 135 (2007), no. 9, 2877-2886.

[5] Duren, P.L., Univalent functions, Grundlehren der Mathematischen Wissenschaften 259, Springer-Verlag, New York, 1983. MR708494 (85j:30034)

[6] FitzGerald, C.H., Horn, R.A., On quadratic and bilinear forms in function theory, Proc. London Math. Soc. (3) 44 (1982), no. 3, 554-576. MR656249 (83g:30039)

[7] Gantmacher, F.R., The Theory of Matrices (Vol. 2), Chelsea, New York, 1989.

[8] Garcia, S.R., Conjugation and Clark operators, Contemp. Math. 393 (2006), 67-112. MR2198373

[9] Garcia, S.R., Putinar, M., Complex symmetric operators and applications, Trans. Amer. Math. Soc. 358 (2006), 1285-1315. MR2187654 (2006j:47036)

[10] Garcia, S.R., Putinar, M., Complex symmetric operators and applications II, (to appear: Trans. Amer. Math. Soc.) MR2187654 (2006j:47036)

[11] Halmos, P.R., A Hilbert Space Problem Book (Second Edition), Springer-Verlag, New York, 1982. MR675952 (84e:47001)

[12] Hasanov, V.I., An iterative method for solving the spectral problem of complex symmetric matrices, Comput. Math. Appl. 47 (2004), 529-540. MR2051327 (2005c:65031)

[13] Horn, R.A., Johnson, C.R., Matrix Analysis, Cambridge Univ. Press, Cambridge, 1985. MR832183 (87e:15001)

[14] Howle, V.E., Vavasis, S.A., An iterative method for solving complex-symmetric systems arising in electrical power modeling, SIAM J. Matrix Anal. Appl. 26 (2005), 1150-1178. MR2178216 (2006h:65045)

[15] Jacobson, N., Normal semi-linear transformations, Amer. J. Math. 61 (1939), $45-58$. MR1507359

[16] Nikolski, N.K., Operators, Functions, and Systems: An Easy Reading. Vol. 1: Hardy, Hankel, and Toeplitz. Vol. 2: Model operators and systems, American Mathematical Society, Providence R.I., 2002. MR1864396 (2003i:47001a)

[17] Peller, V.V., Hankel Operators and Their Applications, Springer Monographs in Mathematics, Springer-Verlag, 2003. MR1949210 (2004e:47040)

[18] Reed, M., Simon, B., Methods of Modern Mathematical Physics I: Functional Analysis, Academic Press, 1980. MR751959 (85e:46002)

[19] Reitzinger, S., Schreiber, U., van Rienen, U., Algebraic multigrid for complex symmetric matrices and applications, J. Comput. Appl. Math. 155 (2003), 405-421. MR1984298

[20] Schur, I., Ein Satz über Quadratische Formen mit Komplexen Koeffizienten, Amer. J. Math. 67 (1945), 472-480. MR0014048 (7:234c)

[21] Scott, N.H., A new canonical form for complex symmetric matrices, Proc. Roy. Soc. London Ser. A 441 (1993), no. 1913, 625-640. MR1225775 (94e:15029)

[22] Scott, N.H., A theorem on isotropic null vectors and its application to thermoelasticity, Proc. Roy. Soc. London Ser. A 440 (1993), no. 1909, 431-442. MR1232840 (94e:15020)

[23] Siegel, C.L., Symplectic geometry, Amer. J. Math. 67 (1943), 1-86. MR0008094 (4:242b)

[24] Takagi, T., On an algebraic problem related to an analytic theorem of Carathéodory and Fejér and on an allied theorem of Landau, Japan J. Math. 1 (1925), 83-93.

[25] Wellstein, J., Über symmetrische, alternierende und orthogonale Normalformen von Matrizen, J. Reine Angew. Math. 163 (1930), 166-182.

Department of Mathematics, Pomona College, 610 North College Avenue, ClareMONT, CALIFORNia 91711

E-mail address: Stephan.Garcia@pomona.edu

URL: http://pages . pomona.edu/ sg064747 